\title{
An empirical analysis of Brazilian courts law documents using learning techniques
}

\author{
Bruno dos Santos F. Silva, Marjory da Costa-Abreu \\ ${ }^{1}$ Departamento de Informática e Matemática Aplicada (DIMAp) \\ Universidade Federal do Rio Grande do Norte (UFRN) \\ Natal - RN - Brazil \\ bruno@ppgsc.ufrn.br, marjoryedimap.ufrn.br
}

\begin{abstract}
This paper describes a survey on investigating judicial data to find patterns and relations between crime attributes and corresponding decisions made by courts, aiming to find import directions that interpretation of the law might be taking. We have developed an initial methodology and experimentation to look for behaviour patterns to build judicial sentences in the scope of Brazilian criminal courts and achieved results related to important trends in decision making. Neural networks-based techniques were applied for classification and pattern recognition, based on Multi-Layer Perceptron and Radial-basis Functions, associated with data organisation techniques and behavioral modalities extraction.
\end{abstract}

\section{Introduction}

There are currently around 90 million lawsuits in Brazil [CNJ 2018] and, although electronic virtualisation of the judicial process already covers a great part of those cases, there is still a huge challenge to streamline the process, analyse and judge each of the cases individually. Facing this scenario, it is important to search for innovative work processes and tools that can potentially help in the continuous improvement of judicial activity.

Meanwhile, machine learning has evolved considerably in use of intelligent data processing techniques [Haykin 2001, Goodfellow et al. 2016], which allows generalisation of knowledge, natural language recognition and machine learning from samples, associated to emerging commercial products adopting intelligent technologies in different application contexts (eg. social networks [Wang 2010], image recognition [Srivastava et al. 2015], automatic translation [Branting 2017], text processing [Aletras et al. 2016], among others), produces a suitable scenario for study and experimentation of such applications in Law area.

The application of intelligent techniques for machine learning as facilitator of the decision-making process of magistrates of Brazilian judiciary could increase productivity of Courts [Coelho 2017], considering capacity of processing legislation and documents that would be dedicated to computer [Thammaboosadee et al. 2012], and would give greater equal treatment of cases (isonomia in Brazilian Portuguese), since it would replace the human components of overload and absorption [Fonseca et al. 2018]. There is a lot of research and projects to deliver intelligent products into the judicial process as [Thammaboosadee et al. 2012, Fersini et al. 2009, Branting 2017].

Thus, this work aims to investigate whether it is possible to infer relevant biases in judicial sentences performed by magistrates using mathematical modelling of data, find 
disruptive relations between crime attributes extracted from real cases and corresponding decisions, and also searches for biometric behavioural modalities. Samples used in this experimentation were collected from real lawsuits by Law researchers, translated in normalised numerical data accord to their meaning and used to train machine learning models.

This paper is organised as follows. Section 2 shows relevant works and related techniques about how to transfer knowledge to computer systems and their application in Law. Section 3 presents how data was used and architectural decisions about neural network models used. Section 4 presents how far we have achieved in this work. Finally, we conclude the paper and suggest further work for automation in judicial sentencing analyses.

\section{Machine Learning and Law}

Using intelligent techniques, such as machine learning, has been an investment target to legal institutions - and associated researchers - as this became a solution to automatic manipulation of judicial processes [Coelho 2017, Grimm 2006, Borges et al. 2003], duo to large volume of lawsuit processes in court houses [CNJ 2018] or legislation and jurisprudence used for legal disputes by lawyer offices [Giuseppe Contissa 2018]. These techniques suggest some compliance to law-labor model, which involves data classification, patterns identification and decision making [Aikenhead 1996] based on previous examples and previously classified standards, brings these technologies into a convincing ally to the management of the judiciary [Maranhão 2018].

Artificial neural networks (ANNs) are the main artificial intelligence technique to machine learning [Goodfellow et al. 2016]. They are inspired by human neurological cells, translated to mathematical models and usually are organized in several layers, some algorithmic control of learning and other tuning parameters. ANNs were defined from McCulloch-Pitts work, evolved to Perceptron model and consequent multi layer Perceptron (MLP) model, which achieved classification of complex linearly divided problems domain. All of this structure trained using an error backpropagation algorithm also known as least minimum square (LMS) algorithm [Haykin 2001].

An alternative to MLP neural networks are Radial-Basis Functions (RBF) neural networks [Orr 1996]. This model is built with two layers of units, using radial activation function in hidden and some linear function in output. This model has some advantage over MLP networks for classification, as its ability to approximate each part of a domain, but not for series forecasting - which is not a goal for this work.

From technological perspective, a lot of work has been done bringing machine learning to end-users, in smartphones and social media like Google ${ }^{1}$ and Facebook ${ }^{2}$, to students and scientists, like Wolphram ${ }^{3}$, etc. Also for Law, products made using machine learning techniques as Evisort ${ }^{4}$ - doing automatic categorisation of contracts and related

\footnotetext{
${ }^{1}$ https://ai.google/

${ }^{2}$ https://research.fb.com/category/facebook-ai-research/

${ }^{3}$ https://www.wolframalpha.com/

${ }^{4}$ https://evisort.com/
} 
laws, LawGeex ${ }^{5}$ - allowing automatic contract reviews and suggestions - and RavelLaw ${ }^{6}$ - for legal search, analytics, and visualization.

There is a lot of work done or in progress in the literature about use of AI in Law, as [Giuseppe Contissa 2018] made an overview about changes and challenges to using AI in Law, from expert systems to machine learning and big data, or [Aikenhead 1996] discusses successful and potential experiences regarding the use of ANNs to mapping concepts/attributes present in case decisions, know as Theft Act 1968 (England) ${ }^{7}$, and AI Now[AINow 2018] presents its main concerns about the use of automated decisionmaking systems in US Courts and core social institutions, compiling activities about use of machine decision in areas such medicaid, government benefits, criminal risk assessment, criminal DNA analysis and some others.

Despite the existence of several works in the area, we did not find any published work that made a contextual analysis of how to apply the parameters used by magistrates for law enforcement and their decisions. This approach would allow evaluating the method adopted, the quality of the grounds and its consequences for the defendants, as a way of monitoring and self-assessment of the legal work.

\section{Methodology}

Data used for this study were obtained by the research project "Além da Pena", which consists of scientific action to promote the conversation between the proposed law and presupposed law, which through empirical investigations investigates patterns or interconnections present in the criminal sentences[Saboya 2014].

Researchers have carried out an inventory of real judicial processes that have been processed in the Brazilian judiciary in last decade, performing annotations in criminal prosecutions data from entry to trial, within delimited parameters provided for by the penal code, observed and necessary to compose a judicial process of that type, with specific metrics capable of normalisation. This procedure, for lawsuit data tabulation, is not regularly available in electronic processes systems analysed, but these work allowed its mathematical analysis, using intelligent or even statistical techniques, in search of implicit relationships hidden by the practice of law enforcement.

Attributes used for description of criminal proceedings include the office case file, the reference year, the gender of the accused, if there has been a confinement and / or detention, the number of days imposed as a penalty of detention or imprisonment, if there was a fine and how many days of a fine, what is the initial compliance regime, what criteria were adopted for establishing the penalty (Art 59 of Federal Law 2848/1940)[CongressoNacionalBrasileiro 1940], among others, distributed in more than 30 attributes being studied. These attributes are listed in Table 1.

There were 463 lawsuit cases collected, distributed randomly between regions and years from last decade, using cases where accused were men or women, with penalties of detention or imprisonment, treated by different judges, all of them compiled by a small group of researchers. These attributes are used regularly and forced by law by courts to

\footnotetext{
${ }^{5} \mathrm{https}: / / \mathrm{www} . l$ lawgeex.com/platform/

${ }^{6} \mathrm{https}: / /$ home.ravellaw.com/

${ }^{7}$ https://www.legislation.gov.uk/ukpga/1968/60/contents
} 


Office case file
Judge
Gender
Days of imprisonment
Background
Detention
Personality
Circumstances
Days of fine
Victim behavior
Base-penalty
Confession
Minimum penalty
Penalty suspension (sursis)
Judgements moralising
Inconsistent culpability criteria
Inconsistent personality criteria
Ne bis in idem for Art 59 circumstances

Lawsuit number

Year

Imprisonment

Compliance regime

Social conduct

Days of detention

Reasons

Fine

Consequences

Days of reclusion

Recidivism

Penalty redux

Rights restricted conversion

Appel in freedom

$\mathrm{Ne}$ bis in idem for basic circumstances

Inconsistent Social Conduct criteria

Inconsistent Victim's behavior criteria

Table 1. Characteristics of sentences

define guiltiness, penalties, and general criteria.

We have used a normalisation [Dash and Liu 1997, Borges et al. 2003] technique in data as preprocessing requirement to avoid distortions and differences in some of the characteristics represented by numbers (eg. days of imprisonment or days of fine), and based this procedure on Keras ${ }^{8}$ library, which transforms each value to zero to one floatnumbers interval, with zero mean and unit variance scaling.

In this paper, two frameworks were used, the first one based on a Multi Layer Perceptron (MLP) with Backpropagation and a Radial Basis Function (RBF) neural network. For both cases, the attributes for training were submitted to K-fold cross validation technique for re-sampling, due to the limited data sample.

For this sampling strategy, dataset was divided into 10 groups and one of them is used for testing and others for training. After each training cycle another group is used for test, until all 10 groups had been used. As dataset was limited to 463 samples, this technique allowed us to achieve significant results.

For each framework of ANNs, MLP or RBF, dataset used for training was based in looking for patterns in judgements by gender of the accused - despite other relations we should still scavenge on data in further experimentations - i) considering all attributes, and ii) considering 8-attributes from Art. 59 (penalty criteria circumstances) - these restricted subset of parameters is a way of ensure individualization of the sentence while allowing the magistrate to exercise his interpretation of the law within pre-established limits and criteria.

The artificial neural networks environment used to train and evaluate models in

\footnotetext{
${ }^{8}$ https://keras.io/
} 


\begin{tabular}{l|c|c|c|r} 
Inference & Layers & Params & KFold & Accuracy \\
detention & $30-16-4-1$ & Relu-Sigmoid & 30 & $93.55 \%$ \\
imprison & $8-5-16-1$ & Relu-Softmax & 8 & $93.55 \%$ \\
gender & $30-64-8-1$ & Sigmoid & k=5 & $93.55 \%$ \\
gender59 & $8-16-4-1$ & beta=3-Softmax & k=3 & $91.35 \%$
\end{tabular}

Table 2. Training with MLP

this paper were hosted by Google Collaboration Project named Google Colab ${ }^{9}$, as a Jupyter ${ }^{10}$ notebook served by virtual machines with GPU, 12GB RAM and 360GB for storage, also with facilities related to sharing code base on Python ${ }^{11}$ and installed APIs as Keras $^{12}$, Tensorflow ${ }^{13}$, among others.

\section{Results}

Training neural networks proposed in this work was carried out in two stages, one performed by MLP neural network and other using a RBF neural network. In both cases, empirical adjustments were made in architecture until the effective results with convergence indicators, considering precision increase, computational resources used, training and evaluation timing and representative results in domain.

Some adjustments made were related to tuning parameters of model, as number and depth of hidden layers, number of folds used to organised and randomly mixture set of samples available to network training, use of different loss and optimisers (learning algorithm engines) available from Keras framework. For MLP neural networks, architecture presented with better results using two hidden layers, using around 16-20 neurons, sigmoid and linear rectifier (ReLu) loss functions.

For RBF neural networks, best results related to convergence criteria were achieved with 16-25 neurons in hidden layer, also based on sigmoid and ReLu loss functions, and beta parameter set to 3, for learning rate control. Both cases had better results using K-Fold set to five folds.

We have done tests for artificial neural network convergence of learning strategy using inferences about compliance regime (detention or imprisonment), and gender (with all 30 attributes and with subset related to Brazilian Penal Code Art. 59 [CongressoNacionalBrasileiro 1940], that are 8 attributes, which defines criteria for sentencing and consider personal interpretation of judges decision making).

The most expressive result was related to accuracy in decisions classification by defendant's gender for trained cases, even in training using the 8 parameters from Art. 59. In Table 2 were compiled best results for each type of inference, for classification of detention or imprisonment results were insignificant, but for gender classification based in all attributes listed in Table 1 or even in attributes related to Art. 59, results reached accuracy around $91.34 \%$ to $93.55 \%$.

\footnotetext{
${ }^{9}$ https://collab.research.google.com

${ }^{10}$ https://jupyter.org/

${ }^{11}$ https://www.python.org/

${ }^{12} \mathrm{https}: / /$ keras.io/

${ }^{13}$ https://www.tensorflow.org/
} 


\begin{tabular}{l|c|c|c|r} 
Inference & Layers & Params & KFold & Accuracy \\
detention & $30-525-1$ & Relu-softmax & $\mathrm{k}=5$ & $12.52 \%$ \\
imprison & $30-525-1$ & Adam-mse & $\mathrm{k}=10$ & $4.01 \%$ \\
gender & $30-525-1$ & beta=3-Sigmoid & $\mathrm{k}=10$ & $91.34 \%$ \\
gender59 & $8-525-1$ & beta=3-Relu-softmax & $\mathrm{k}=5$ & $91.35 \%$
\end{tabular}

Table 3. Training with RBF

In Table 3 results are presented for similar cases but for Radial-basis functions neural networks, which could confirm similar inferences learned from MLP neural networks, but using different computational resources and algorithms, in this case running in better execution time as compared to the first model.

\section{Conclusion}

Despite huge lawsuit volume in the Brazilian judicial structure and improvements obtained with process virtualisation strategies, use of machine learning and decision-making support software could lead performance improvement for state legal institutions, providing tools for performing data mining, analysis and classification, and even high-level inference, allowing for faster and fairer procedures.

In this experiment we could identify the correlation between judges decisions based on the gender of the accused, indicating that there is a definite relationship between the criteria for judgment and their status as male or female. Indeed there was a great assertiveness in the identification of a sentence, indicating that depending on the parameters identified in real case, a sentence may be recommended computationally.

Moreover, in this experiment, we observed that there are behavior patterns in judges that may indicate the existence of work-overload influencing act of judgment, or adoption of judgment standards based on tendentious perceptions according to the social group. Other inferences could be achieved through deep analysis of data of evidences, mixed with crime attributes. These inferences drawn from machine learning should contribute to further discussion of Law area, whether in the scope of the "Além da Pena" project or other research groups, including defendant's lawyers and crime science forecasting.

Machine learning framework used in this work was based on common artificial neural network architectures suitable for the task of data classification and pattern identification, but using input data manually compiled, which should not be available in actually used judicial systems. This scenario must be adapted to a deeper integration with process virtualisation framework, to turn this solution useful and realistic.

\section{References}

Aikenhead, M. (1996). Uses and abuses of neural networks in law, the. Santa Clara Computer \& High Tech. LJ, 12:31.

AINow, A. N. I. (2018). Ai now report 2018. Technical report.

Aletras, N., Tsarapatsanis, D., Preoţiuc-Pietro, D., and Lampos, V. (2016). Predicting judicial decisions of the european court of human rights: a natural language processing perspective. PeerJ Computer Science, 2:e93. 
Borges, F., Borges, R., and Bourcier, D. (2003). Artificial neural networks and legal categorization. In The 16th Annual Conference on Legal Knowledge and Information Systems (JURIX'03), page 187.

Branting, K. L. (2017). Automating judicial document analysis. In Proceedings of the Second Workshop on Automated Semantic Analysis of Information in Legal Text (ASAIL 2017), London, UK. CEUR.

CNJ, C. (2018). Justiça em números - analytical report 2018. Technical report. baseline 2017.

Coelho, J. V. d. A. B. R. (2017). Aplicações e implicações da inteligência artificial no direito.

CongressoNacionalBrasileiro (1940). Decreto-lei no 2.848, de 07 de dezembro de 1940.

Dash, M. and Liu, H. (1997). Feature selection for classification. Intelligent Data Analysis, 1(1):131 - 156 .

Fersini, E., Messina, E., Arosio, G., and Archetti, F. (2009). Audio-based emotion recognition in judicial domain: A multilayer support vector machines approach. In International workshop on machine learning and data mining in pattern recognition, pages 594-602. Springer.

Fonseca, F. F., Cunha, D. M., Vieira, E. O., and Modena, C. M. (2018). Implicações de novas tecnologias na atividade e qualificação dos servidores: Processo judicial eletrônico e a justiça do trabalho. Revista Brasileira de Saúde Ocupacional, 43:1-12.

Giuseppe Contissa, Francesca Lgioia, M. L. H.-W. M. (2018). Towards consumerempowering artificial intelligence. In Twenty-Seventh International Joint Conference on Artificial Intelligence (IJCAI-18), pages 5150-5157.

Goodfellow, I., Bengio, Y., and Courville, A. (2016). Deep Learning. MIT press.

Grimm, C. (2006). Dosimetria da pena utilizando redes neurais.

Haykin, S. (2001). Redes neurais: princípios e prática. Bookman Editora, 2nd edition.

Maranhão, J. (2018). O impacto na justiça. In Yoshida, E., editor, EXAME CEO - Inteligência Artificial, pages 80-83. Editora Abril.

Orr, M. J. L. (1996). Introduction to radial basis function networks.

Saboya, K. (2014). Ne bis in idem em tempos de multiplicidades de sanções e de agências de controle punitivo. Jornal de Ciências Criminais, 1:71-92.

Srivastava, N., Mansimov, E., and Salakhutdinov, R. (2015). Unsupervised learning of video representations using lstms. CoRR, abs/1502.04681.

Thammaboosadee, S., Watanapa, B., and Charoenkitkarn, N. (2012). A framework of multi-stage classifier for identifying criminal law sentences. volume 13, pages 53 - 59. Proceedings of the International Neural Network Society Winter Conference (INNS-WC2012).

Wang, A. H. (2010). Detecting spam bots in online social networking sites: A machine learning approach. In Foresti, S. and Jajodia, S., editors, Data and Applications Security and Privacy XXIV, pages 335-342, Berlin, Heidelberg. Springer Berlin Heidelberg. 